\title{
Keeping HSV-1 dormant
}

Following host infection, herpes simplex virus 1 (HSV-1) remains latent in peripheral neurons. $\beta$-nerve growth factor (NGF) was known to have a role in maintaining HSV-1 latency, but the molecular details had not been described. Now, Camarena et al. dissect the host signalling pathway that is activated by NGF and that supports HSV-1 latency.

As NGF activates two receptors, the tyrosine kinase TrkA and p75 neurotrophin receptor, the authors first sought to identify which receptor is involved in maintaining HSV-1 latency. Inhibition of TrkA, but not p75, in a neuronal cell culture model induced HSV-1 reactivation, as detected using a green-fluorescent protein (GFP)tagged reporter. This suggests that NGF interacts with TrkA to maintain latency.

Binding of NGF to TrkA is known to activate several host cell signalling pathways, including the mitogenactivated protein kinase (MAPK) and phosphoinositide 3-kinase (PI3K) pathways. The authors observed that chemical inhibition of PI3K signalling, but not MAPK or phospholipase C $\gamma$ signalling, induced HSV-1 reactivation in the neuronal cell culture model. Furthermore, HSV-1 reactivation depended specifically on inhibition of the p110 $\alpha$ subunit of PI3K and not the $\mathrm{p} 110 \beta$ or $\mathrm{p} 110 \delta$ subunits.

One of the downstream effects of PI3K-signalling activation is the recruitment of 3-phosphoinositidedependent protein kinase 1 (PDK1) to the plasma membrane. Chemical inhibition of PDK1 or treatment with small hairpin RNAs specific for PDK1 led to HSV-1 reactivation in neuronal cultures to levels comparable to those observed with the PI3K inhibitor. PDK1 is known to phosphorylate the serine/threonine kinase Akt, and chemical inhibition of Akt led to high levels of HSV-1 reactivation. Together, these findings show that, downstream of NGF binding to TrkA, HSV-1 latency is maintained by PDK1-mediated phosphorylation of Akt.

Interestingly, different growth factors that activate PI3K signalling showed different abilities to induce HSV-1 latency in the neuronal cell culture. Glial cell line-derived neurotrophic factor (GDNF) showed some capacity to suppress HSV-1 reactivation when NGF was absent from the culture medium, albeit not as great a capacity as NGF, and epidermal growth factor (EGF) had no effect. This was due to differences in the kinetics of Akt phosphorylation. Specifically, NGF induced sustained Akt phosphorylation, EGF induced a short-lived increase in Akt phosphorylation and GDNF showed intermediate phosphorylation kinetics. It therefore seems that prolonged signalling through Akt phosphorylation regulates HSV-1 latency.

So, HSV-1 latency is maintained in peripheral neurons through sustained PI3K signalling induced by binding of NGF to TrkA. This activates the p110a PI3K subunit, leading to PDK1 activation and Akt phosphorylation. Further studies should now seek to determine how viral factors involved in latency interact with the neuronal factors activated by PI $3 \mathrm{~K}$ signalling.

Rachel David, Assistant Editor, Nature Reviews Microbiology

ORIGINAL RESEARCH PAPER Camarena, V. et al. Nature and duration of growth factor signaling through receptor tyrosine kinases regulates HSV-1 latency in neurons. Cell Host Microbe 8 , 320-330 (2010) 